\title{
An automatic screening method for primary open-angle glaucoma assessment using binary and multi-class support vector machines
}

\author{
Pikul Vejjanugraha $^{\mathrm{a}, \mathrm{b}, *}$, Waree Kongprawechnon ${ }^{\mathrm{a}}$, Toshiaki Kondo ${ }^{\mathrm{a}}$, Kanokvate Tungpimolrut ${ }^{\mathrm{c}}$, \\ Kazunori Kotani ${ }^{\text {b }}$ \\ a School of Information, Communication, and Computer Technologies, \\ Sirindhorn International Institute of Technology, Thammasat University, Khlong Luang, \\ Pathum Thani 12120 Thailand \\ b School of Information Science, Japan Advanced Institute of Science and Technology, Nomi, \\ Ishikawa 923-1211 Japan \\ c National Electronics and Computer Technology Centre, \\ National Science and Technology Development Agency, Khlong Luang, Pathum Thani 12120 Thailand
}

${ }^{*}$ Corresponding author, e-mail: pikulvej@jaist.ac.jp

Received 7 Mar 2017

Accepted 8 Aug 2017

\begin{abstract}
Glaucoma is a chronic progressive eye condition leading to permanent visual loss. An automatic screening system is necessary to detect primary open-angle glaucoma because it is an insidious disease appearing without symptoms or early warning signs. This work introduces an automatic screening technique to diagnose glaucoma using a support vector machine (SVM). Two case studies are investigated: binary-stage and multi-stage classification of glaucoma. First, there is a comparison of the performance of the hard threshold-based approach to the supervised learning approach using an SVM. Image segmentation techniques are performed to detect important features: the actual sizes of the optic cup and optic disc in vertical and horizontal directions. SVMs with a linear kernel function are used to generate the classifier model, and the results show that using threshold-based classification is inadequate to screen glaucoma. In a second case study, an SVM is applied to develop the classification algorithm focused more on the detection of the glaucoma suspect stage, which is an intermediate stage between the healthy and glaucoma stages. A polynomial kernel function is used to implement the classification model. The unbalanced decision tree (UDT) and one-versus-the-rest (OVR) techniques are combined in the models in order to overcome the limitations of an SVM. Finally, the combination of an SVM with both UDT and OVR techniques yields a reliable result with respect to belonging classes at $99.4 \%$.
\end{abstract}

KEYWORDS: cup-to-disc ratio

\section{INTRODUCTION}

Glaucoma is a group of irreversible eye diseases without early warning sign which causes progressive damages to the optic nerve head (ONH). Early diagnosis and treatment can help to preserve the eyesight. Glaucoma can be divided into two main types: open angle glaucoma (OAG) and closed angle glaucoma. OAG is the most common type of glaucoma worldwide. This work focused on an automatic screening technique to diagnose OAG into several stages. The suspect stage is important because a patient will receive a warning and treatment before it progresses and shows symptoms such as headache from abnormal pressure inside the eyeball. In clinical examination, the intraocular pressure is tested first. After that a fundus image is taken to observe some abnormalities in the retina. This provides important information to extract, such as the shape and asymmetry of the optic disc (OD), size and depth of the optic cup (OC), vertical cupto-disc ratio ${ }^{1-3}$, nerve fibre layer abnormalities, and peripapillary atrophy. If some abnormalities appear, visual field loss is determined. This can appear in one or both eyes. These abnormalities might be caused by many factors, but glaucoma is one of the risk factors that damages the $\mathrm{ONH}$ and gradually leading to a loss of vision. In the rural area of Thailand, there are shortage of ophthalmologists, technicians, healthcare workers, and early treatment instruction. This system would help reduce the gap between those problems by providing an 
automatic screening system to diagnose the disease based on a supervised learning technique.

For the supervised learning technique, a feature of the target class must be extracted in order to generate a decision function or model to classify each stage of the disease. In this work, the OD and OC segmentations are considered. There are several techniques provided during previous work. For the preprocessing process, the colour space of the retinal image can be considered in order to obtain some important information such as RGB ${ }^{3,4}$, $\mathrm{CYM}^{5}$. Each band provides different information of the object, e.g., $\mathrm{G}$ band of RGB and $\mathrm{M}$ band of CYM provide the OC information better than the others. Some of them consider appearancebased techniques or model-based techniques, for example, the super pixels technique 6,7 , active contour $\operatorname{model}^{7,8}$, Hough transform ${ }^{6,9}$, and template matching ${ }^{4}$. In some cases, in the OC there is an occlusion problem from the blood vessels which can be overcome by smoothing the boundary by ellipse fitting techniques. However, in some purpose, the region or area of the OC can be detected by a region of support concept ${ }^{10}$ in order to derive the desired OC boundary. The texture and intensity features ${ }^{11}$ can be used as well. The intensity features are detected by wavelet-based analysis and local binary pattern. The correlation or similarity of the features should be evaluated in order to reduce the redundant features. The dimension of the features can be reduced by techniques such as principal component analysis and linear stepwise regression. For the classification part, the classifiers that are normally used include $K$-mean, fuzzy $C$ means clustering ${ }^{8,12}$, Nave Bayesian technique ${ }^{13}$, neural network $(\mathrm{NN})^{8,14}$, support vector machine $(\mathrm{SVM})^{5,14-16}$. Among these, NN and SVM provide high performance and robustness for higher dimensional classification.

The ratio of OC to OD in the vertical direction (CDRv) is considered an important feature to check the abnormality of a retina using a fundus image. Furthermore, the rim-to-disc ratio ${ }^{3}$ was also proposed to be considered for special cases with large OC and OD but healthy rim tissues. Using only $\mathrm{CDRv}$ as a threshold to indicate glaucoma and nonglaucoma linearly is inadequate because there are overlapping values, which have to be analysed in a higher dimensional space. For example ${ }^{17}, \mathrm{CDRv}$ at 0.65 as a threshold value to indicate glaucoma and non-glaucoma provides $80 \%$ accuracy, or 21 false negative (FN) cases and 3 false positive (FP) cases. This work uses the diameters of OC and OD and the
CDR in both vertical (CDRv) and horizontal (CDRh) directions. There are two case studies. In case study A, a comparison among previous work ${ }^{17}$ and the proposed techniques are discussed. The FN and FP have to be reduced, therefore, SVM is introduced in a classification process, diagnosed into two classes: healthy and glaucoma. In case study B, the suspect stage of glaucoma is added. It is the stage between the normal and glaucoma classes. Early detection can be detected based on this suspect class. Multistage classification is applied by combining the SVM with OVR and unbalanced decision tree (UDT) techniques. Five models are generated and considered for the recall and precision of each module. It is found that combining SVM with both OVR and UDT provided the best result at $99.4 \%$, using 10 -fold cross validation. The number of false detections is also an important parameter to analyse the classification performance. The polynomial degree three, kernel function of SVM is selected to generate the decision module that can reduce the number of false detections.

This paper is organized as follows. The materials and methods section provide necessary information about data collection and experimental settings and describes the method. The next section evaluates the results of both case studies. Then, the discussion section interprets and analyses the output information, including the summary of this study.

\section{MATERIALS AND METHODS}

The input data (called fundus images) are obtained from Mettapracharak (Wat Rai Khing) Hospital. A fundus image illustrates an interior lining of the eyeball, including the retina, OD, macula, and the retinal nerve fibre layer. Common diseases of the eye can be initially detected by analysing those components. Thus the fundus image, taken by a Non-Mydriatic Auto Fundus Camera (NIDEX AFC203), is mostly used as a first step during routine medical examination. The NIDEX AFC-203 camera supports high quality images by supplying full frame $(35 \mathrm{~mm} \times 24 \mathrm{~mm}$ ) in a 45-degree field of view. Three ophthalmologists classified the data to normal, suspect, and abnormal classes manually, and the majority-selected technique among the three ophthalmologists is considered as the ground truth. The correlation coefficient matrix is

$$
\left[\begin{array}{ccc}
1 & 0.463 & 0.493 \\
0.463 & 1 & 0.549 \\
0.493 & 0.549 & 1
\end{array}\right]
$$



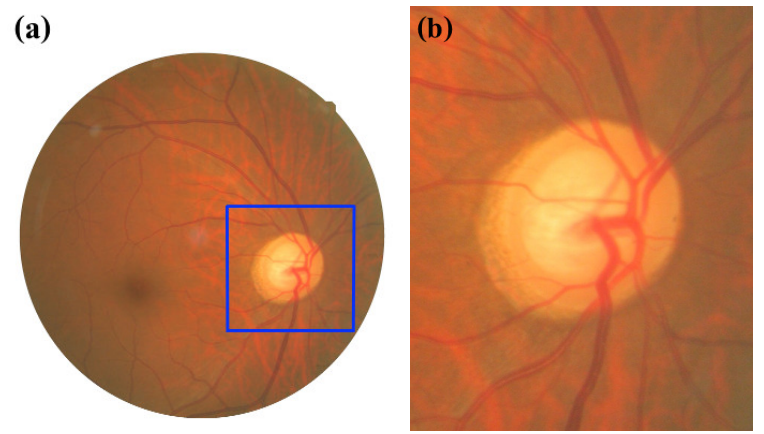

Fig. 1 An input image; (a) the original fundus image and (b) the region of interest.

where the range of $0.4-0.7$ is considered as moderately correlated. The clinical results also widely vary based on their experiences and other considering factors such as age, peripapillary atrophy, blood vessel appearance. The dataset for case study A consists of 60 samples of normal eyes and 60 samples of glaucoma eyes. In case study B, we provide more data in suspect cases and rearrange the overall dataset into three groups based on the ground truth. We provide 60 samples of normal eyes, 50 samples of suspect eyes, and 60 samples for glaucomatous eyes. In the programming part, MATLAB 2013b is used to analyse the system. An algorithm is developed using image processing and statistics and machine learning toolboxes.

\section{Image processing}

To reduce the false detections and computational time, preprocessing is used. The size of the original fundus image is $2912 \times 2912$ pixels in RGB at 72 DPI. These fundus images are saved as JPEG flies. The image resolutions are reduced to 36 DPI in order to reduce the time consumed. Then, to decrease the false detections, the limited area around the OD is set to the region of interest (ROI). The centroid of the ROI is located at the centre of the OD by using the intensity-weighted centroid approach ${ }^{17}$. Then we extend the height and the width from the centroid to create a rectangular ROI with size $475 \times 550$ pixels (Fig. 1 ).

The ROI, $475 \times 550$ pixels, is found for the segmentation process. Since RGB components contain different information, they are separated into red, green, and blue channels. The red band (Fig. 2a) and green band (Fig. 2b.) are selected to detect the $\mathrm{OD}$ and $\mathrm{OC}^{3,4}$, respectively. These separated components can help in detecting the OD and OC more clearly than in grey scale images. To standardize the

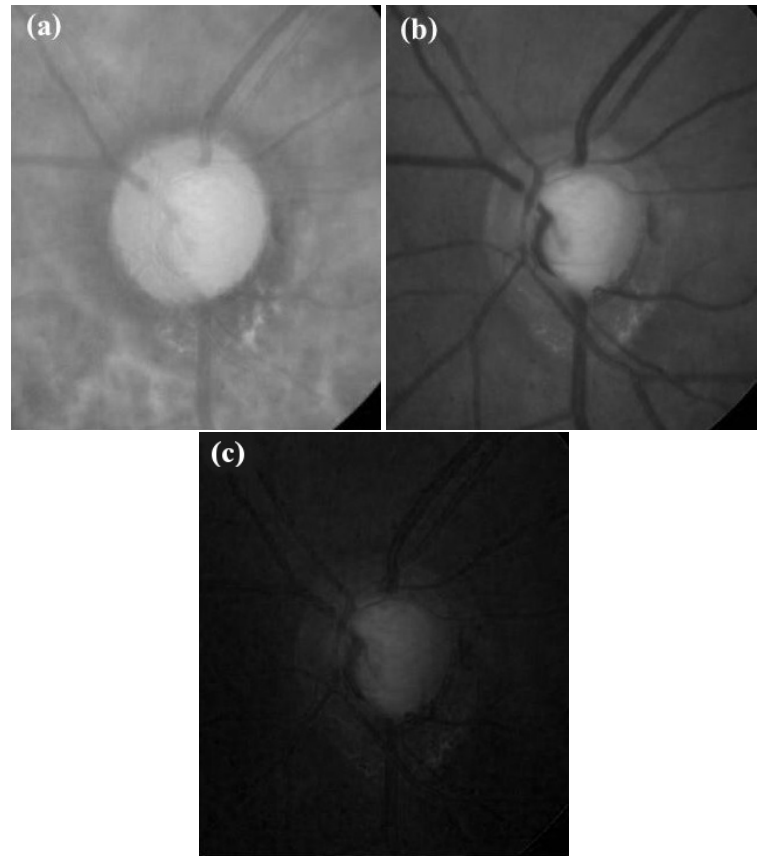

Fig. 2 The RGB components of the fundus image; (a) red component, (b) green component, and (c) blue component.

data, the maximum and minimum values are used to unify the data.

Unwanted noise and blood vessels are removed by a morphological technique and median filter. A closing operation, with disc shape of the structural element, is able to remove background noise inside the OD and the blood vessels. The median filter is applied to smoothen the intensity. Image enhancement is done by a power law transformation. The level-set thresholding with Otsu's method ${ }^{18}$ is performed to extract the OD from the background and turns the grey scale image [0-255] into a black and white image $[0,1]$. Finally, the Canny edge detection is applied to extract the shape of the OC and OD.

To detect the OD, the red component is considered (Fig. 3b). The unwanted noise and blood vessels are removed. Then it is enhanced by the power law transformation technique. The powerlaw component is set to 3 , which makes the image become darker and enhances the brightness of the region of interest (Fig. 3e). Otsu's global threshold is used to detect the brightness area, representing the OD region.

To detect the OC, the green components are commonly considered (Fig. 4b). According to the experimental result, it is shown that the green chan- 


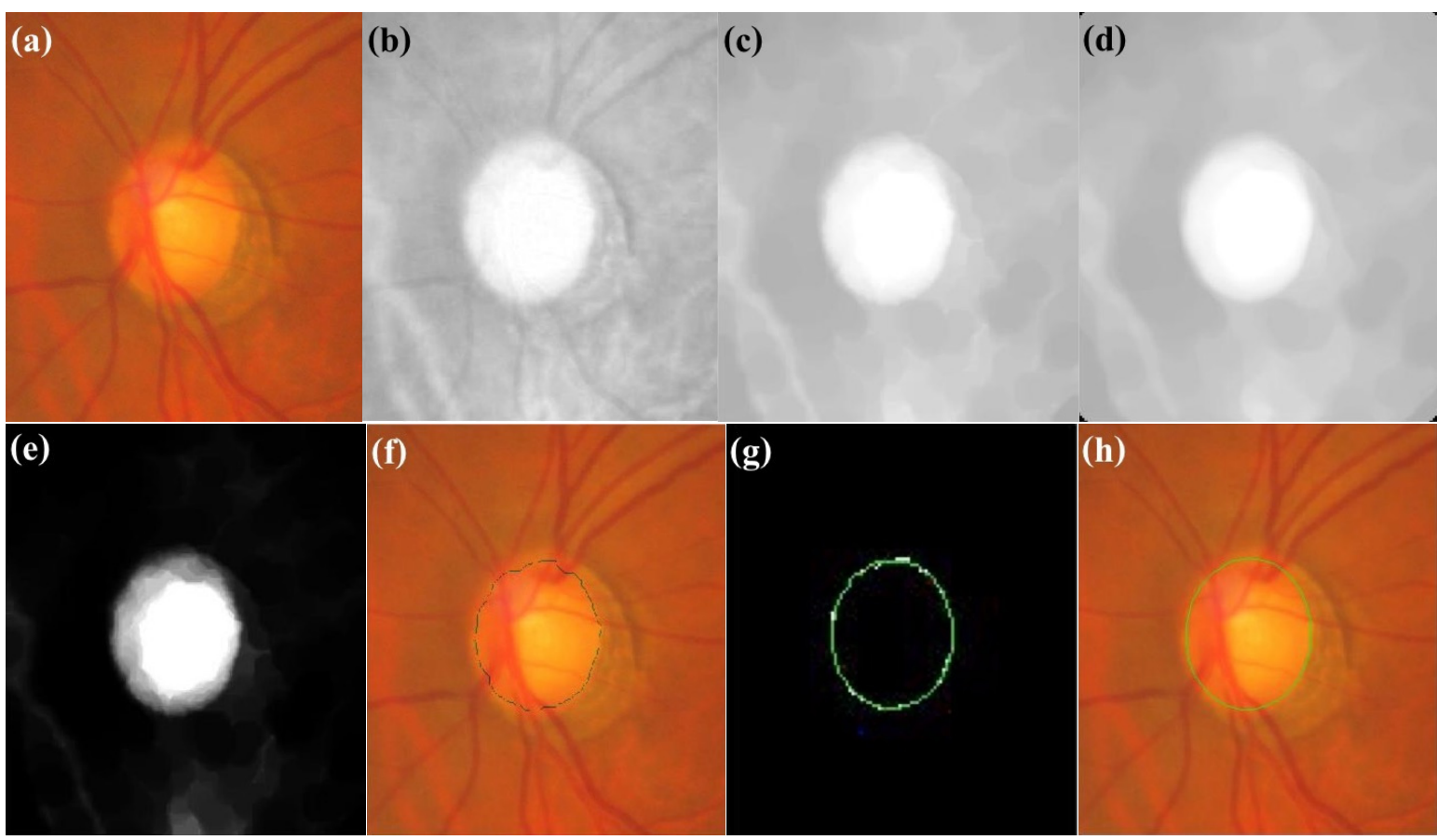

Fig. 3 Optic disc detection; (a) ROI image, (b) red component of RGB image, (c) and (d) perform closing operation and medium filter, (e) apply power law transformation, (f) apply edge detection, ( $g$ ) smoothen by ellipse fitting, and (h) detect the optic disc.

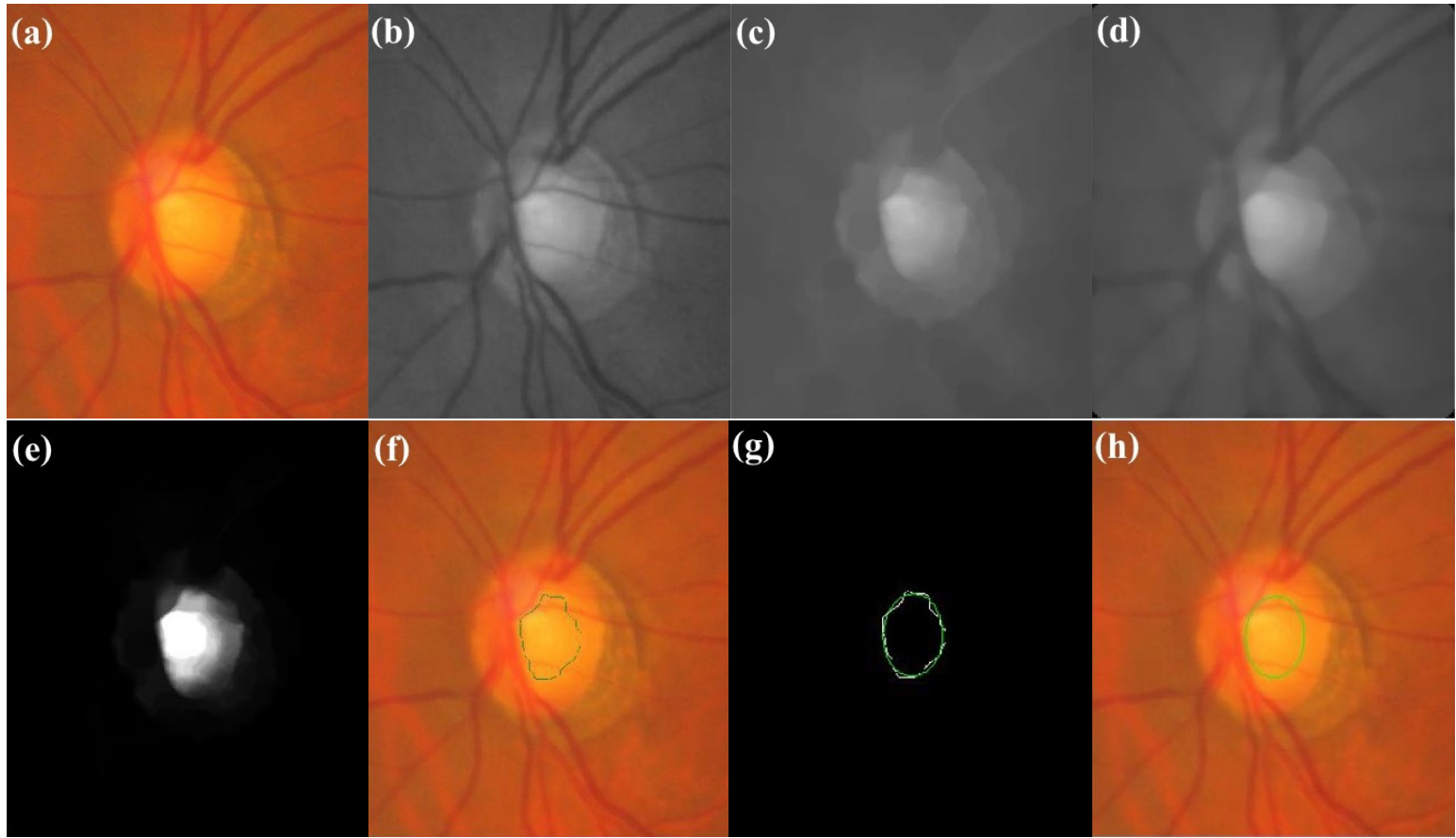

Fig. 4 Optic cup detection; (a) ROI image, (b) green component of RGB image, (c) and (d) perform closing operation and medium filter, (e) apply power law transformation, (f) apply edge detection, ( $g$ ) smoothen by ellipse fitting, and (h) detect the optic cup. 
nel provides a better result than the blue one. The green channel, therefore, is chosen to detect an OC that has more complexity than the OD. The other processes are repeatedly done, following the image segmentation and feature extraction steps. However, there is a difference in the threshold-based function. The intensity of the $\mathrm{OC}$ is brighter than the $\mathrm{OD}$ and the outer regions in the ONH. Hence onethird (brightness) of the threshold level is detected as the OC region. After that, the edge detection is applied to extract the OC boundary.

The power-law $(\gamma)$ transformation is selected to increase the contrast of the region of interest. This image enhancement technique is described as $Y=K X^{\gamma}$, where $Y$ indicates an enhanced output, $X$ is an input, $K$ is a constant, and $\gamma$ is the powerlaw component. When the $\gamma$ is higher than one, the background becomes darker and the object brighter. The blight region (especially the OC) is visualized by mapping a wider range of dark input to the narrow range. In this study, the power-law component $\gamma$ is set to three. To extract useful information, clustering and ellipses fitting techniques are applied. $K$-mean clustering is performed in order to reduce the noise in the boundary from the edge detection. $K$-means clustering is a method of cluster analysis, which aims to partition $i$ observations into $K$ clusters, in which each observation belongs to the cluster with the nearest mean. The clustering mainly considers on the distance from the centroid of ONH to the whole vicinity of ROI. The distance of each data point is calculated as $\sqrt{\left(X_{\text {centre }}-X_{i}\right)^{2}+\left(Y_{\text {centre }}-Y_{i}\right)^{2}}$ to indicates the distance from the data point $\left(X_{i}, Y_{i}\right)$ to the centroid ( $\left.X_{\text {centre }}, Y_{\text {centre }}\right)$ of the ONH. Then the group that contains only OD's boundary is selected and noise is rejected. Ellipse fitting is performed to find the best fit for the boundary of the OC and OD. It is a well-known technique used to smooth the boundary ${ }^{12,14,19}$. It is usually based on a least square fitting algorithm which assumes that the best-fit curve of a given type is the curve that minimizes the algebraic distance over the set of $N$ data points in the least squares sense. It helps to smoothen the OD and $\mathrm{OC}$ boundary after the segmentation technique is completed. Especially in the OC, some parts of its boundary may have occlusion problem with blood vessels.

Fig. 5 shows the input fundus images, Fig. $5 \mathrm{a}$ is the glaucomatous eye and Fig. $5 \mathrm{~b}$ is the nonglaucomatous eye. The difference between the two cases can be observed by the changing size of the bright region inside $\mathrm{ONH}$. When feature extrac-

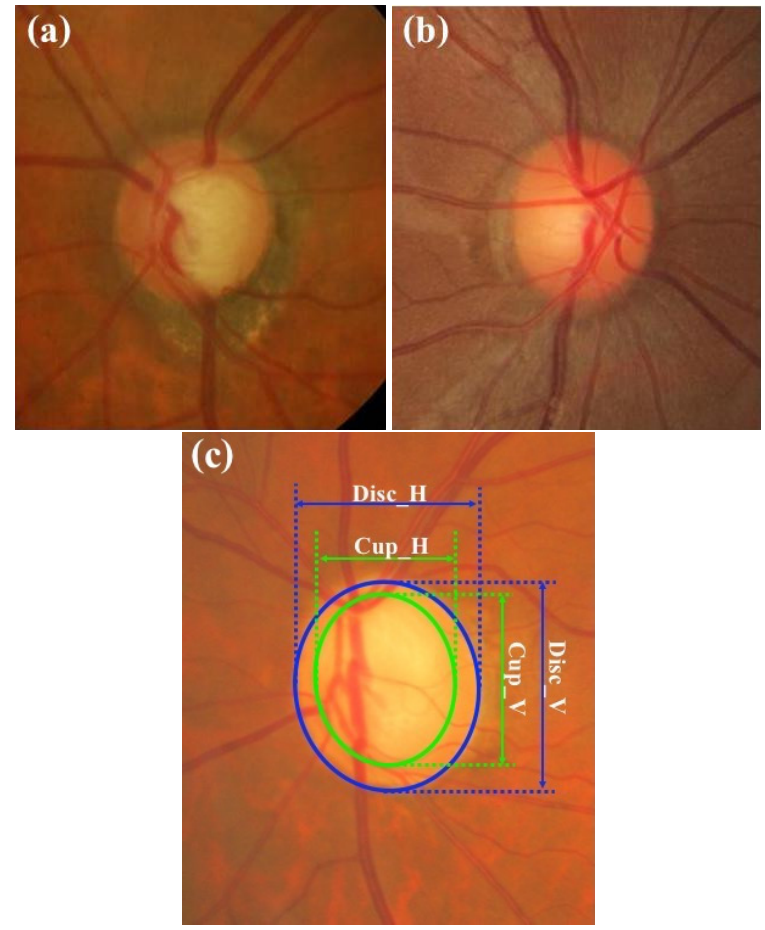

Fig. 5 The input fundus images; (a) glaucomatous eye, (b) normal eye, and (c) the location of the OC and OD in vertical and horizontal directions.

tion is performed, four features from the ellipse fitting technique and two features from CDR calculation are obtained: vertical diameter of an optic cup (Cup_V), horizontal diameter of an optic cup (Cup_H), vertical diameter of an optic disc (Disc_V), horizontal diameter of an optic disc (Disc_H), CDRv, and CDRh (Fig. 5c).

\section{Classification technique}

The classification technique is widely used for a prediction depending on known characteristics learned from the database. An SVM technique is selected as a classifier to find a decision function. It can generate an adaptive decision boundary, based on the distribution of selected information or features. To obtain the feature matrix, ellipse fitting is used to extract the ellipse shape of the OC and OD. Subsequently, the CDR is calculated from the ratio of the OC to the OD. The selection of features is necessary for using the kernel function in order to find the optimal hyper plane for separation of the two classes.

Firstly, binary classification in Case study A is described in detail. An SVM is widely used for classifying a feature in a high dimensional feature space. 
It provides several kinds of kernel functions (decision boundaries) such as linear, polynomial, and radial basis functions. These kernel functions have different characteristics, and the kernel selection depends on the distribution of input information. An SVM with a linear kernel function is selected for Case study A. The SVM transforms the input features in 2 dimensions to a higher dimensional feature space and maximizes the sample distances (support vectors) from the decision hyper plane. To calculate the maximum margin of the support vectors, the kernel function is represented by the Euclidean inner product. The following are expressions of two selected kernel functions, $K_{\mathrm{L}}(x, y)=x^{\mathrm{T}} y$ for linear kernel as default and $K_{\mathrm{P}}(x, y)=\left(x^{\mathrm{T}} y+c\right)^{d}$ for polynomial kernel, where $c \geqslant 0, d$ are parameters that can be adjusted to find the most efficient kernel function. The cost factor for linear and polynomial kernel is set as 1. Based on the experiments in Case study A and B, the weights of the features selected are ranked as follows; CDRv, CDRh, Cup_V, Cup_H, Disc_V, and Disc_H. In Case study A, the parameters for the learning process are defined. First, six features are extracted from 120 training samples. They are separated into two classes and labelled 0 for the target class and 1 for others. An SVM is used and a classifier is generated. The $K$ fold cross validation technique is selected to test the accuracy. $K$ is set to 10 , and then $K-1$ splits are trained. The testing set randomly selected $10 \%$ of the data set. The remaining $90 \%$ are assigned as training data. After the first ten percent is tested, the next testing data is changed to be a new set with no overlap. This procedure is repeated ten times until the last testing set. Then the errors from overall runs are accumulated and described in a confusion matrix.

Secondly, multi classification in Case study B is described by introducing one-versus-the-rest SVM (OVR-SVM) and unbalanced decision tree with SVM (UDT-SVM) in order to overcome a limitation of the traditional SVM, which is effective only in binary classes. The multi-class SVM is used to distinguish three different classes of data, normal 60 samples, suspect 50 samples, and glaucoma 60 samples. The following describes the classification model of each technique. For OVR-SVM (Fig. 6), the data is sent to each subclassifier in parallel. The data set is classified $N$ times in parallel to find the real class to which they belong. The class selection process is done after the results of all classes are tested and evaluated. The highest score is chosen, setting 0 for correct prediction and 1 for incorrect prediction. If

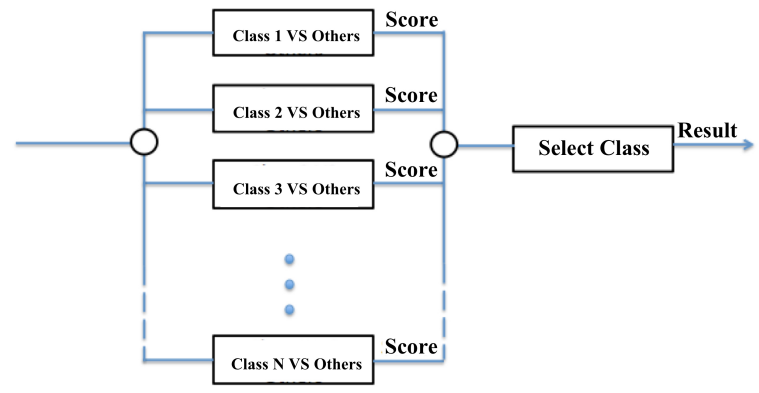

Fig. 6 The architecture of OVR-SVM.

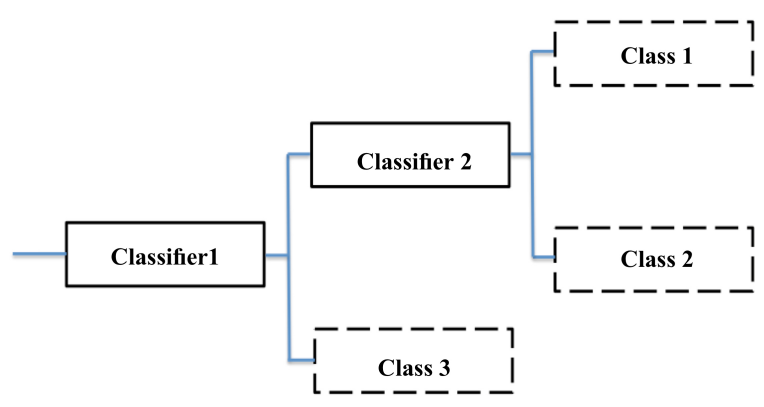

Fig. 7 The architecture of UDT-SVM.

the scores are equal, the nearest mean is used, to analyse which class is closest to the data set.

The UDT-SVM also has a trade off between large and small size of data. For a large size of data, it will eliminate some class of data points for each stage such that all data is trained in the first stage, Classifier 1 (Fig. 7). When glaucoma is classified, the data labelled as Class 3 is removed before applying Classifier 2. After removing data points of Class 3, the remaining data in Classifier 2 is the normal Class 1 and the suspect Class 2 so that the main characters of the normal and suspect classes are differentiated.

\section{RESULTS}

To evaluate the true performance of the system, a 10 -fold cross validation technique is performed on the data set (90\% for training set and 10\% for testing set). True positive (TP) indicates the target class is predicted correctly and true negative (TN) indicates the non-target class is predicted correctly. Classification errors are analysed using confusion matrices and expressed as two different kinds, FP and FN. The abilities of each method are considered by the accuracy (ACC), specificity (SPEC), sensitivity or recall (SENS), and precision (PREC) which are defined as $\mathrm{ACC}=(\mathrm{TP}+\mathrm{TN}) /(\mathrm{TP}+\mathrm{FN}+\mathrm{FP}+\mathrm{TN})$, SENS $=\mathrm{TP} /(\mathrm{TP}+\mathrm{FN})$, SPEC $=\mathrm{TN} /(\mathrm{TN}+\mathrm{FP})$, and 
Table 1 The structure of confusion matrix.

\begin{tabular}{|c|c|c|}
\hline & Predicted target & Predicted non-target \\
\hline Actual target & TP & FN \\
\hline Actual non-target & FP & $\mathrm{TN}$ \\
\hline
\end{tabular}

PREC $=\mathrm{TP} /(\mathrm{TP}+\mathrm{FP})$, where TP is a glaucoma classified as glaucoma, $\mathrm{TN}$ is a healthy person classified as healthy, FP is a healthy person classified as glaucoma, and FN is a glaucoma classified as healthy. FP and FN are parameters for the reliability of the screening system.

In case study B, there is multi-classification and unbalanced data. The data that belong to each class are not equal and the performance measure is different from the binary classification ${ }^{7}$. The weighted average precision $\left(\mathrm{PREC}_{\mathrm{Avg}}\right)$ is used as

$\mathrm{PREC}_{\mathrm{Avg}}=\frac{w_{i-2} \mathrm{PREC}_{i-2}+w_{i-1} \mathrm{PREC}_{i-1}+w_{i} \mathrm{PREC}_{i}}{w_{i-2}+w_{i-1}+w_{i}}$,

where $w_{i}$ represents the number of data points in each class and $i$ is the number of multi-classification classes. The structure of the confusion matrix used in Case study A, described in quantitative terms, is shown in Table 1.

\section{Case study A}

According to previous work ${ }^{17}$, ophthalmologists consider the shape of the OC and OD in the vertical direction (called Cup_V and Disc_V). However, it appears that adding the OC and OD in the horizontal direction (called Cup_H and Disc_H) yields a better result. The three proposed methods are compared for classifying and the number of features.

Method $\mathrm{I}^{17}$. The sizes of the OC and OD are obtained in the vertical direction. The thresholdbased classification is used by considering the value of CDRv. When the CDRv is greater than 0.65, an unhealthy case is identified. Fig. 8 shows the flow chart of this method. After feature extraction, the detected features are directly applied to the threshold-based approach. Application of this technique to our database gives an accuracy of $80 \%$ for all events (Table 2). However, there are $21 \mathrm{FN}$ errors (18\%) and 3 FP errors (2\%). Thus Method II is implemented by adding SVM learning technique to automatically classify glaucoma.

Method II. SVM with three vertical features. Three features are extracted in the vertical direction of the ONH, Cup_V, Disc_V, and CDRv. An SVM is used to separate the glaucoma and non-glaucoma cases. The result is explained in Table 2. Compared

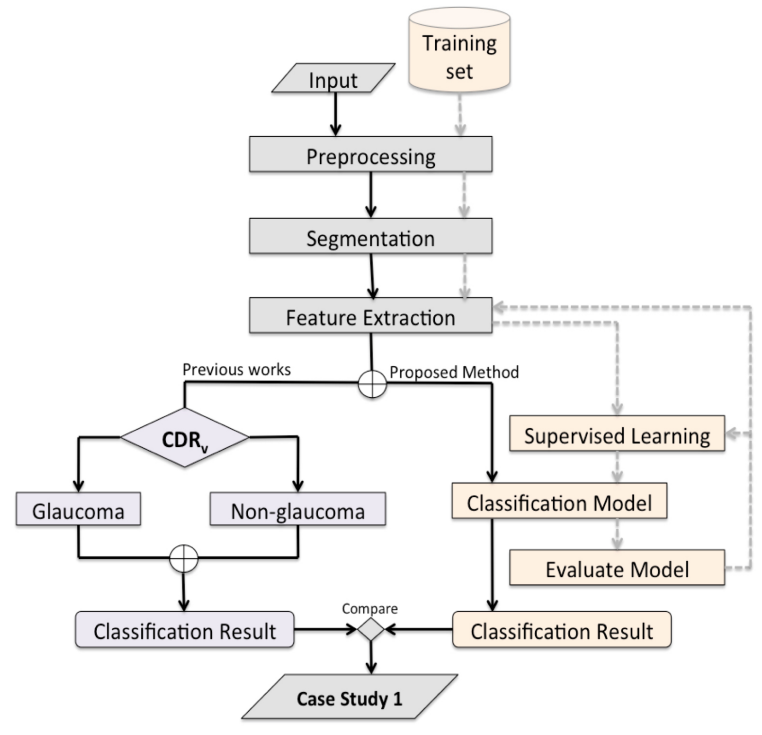

Fig. 8 Case study A.

Table 2 Comparison among three proposed methods.

\begin{tabular}{lccccccc}
\hline & \multicolumn{2}{c}{$\begin{array}{c}\text { Confusion } \\
\text { matrix }\end{array}$} & & \multicolumn{5}{c}{$\begin{array}{c}\text { Classification } \\
\text { performances }\end{array}$} \\
\cline { 2 - 3 } \cline { 6 - 8 } & TP & FN & & SENS & SPEC & PREC & ACC \\
& FP & TN & & & & & \\
\hline Method I & 57 & 21 & & $73 \%$ & $93 \%$ & $95 \%$ & $80 \%$ \\
& 3 & 39 & & & & \\
Method II & 59 & 3 & & $95 \%$ & $98 \%$ & $98 \%$ & $97 \%$ \\
& 1 & 57 & & & & \\
Method III & 59 & 0 & & $100 \%$ & $98 \%$ & $98 \%$ & $99 \%$ \\
& 1 & 60 & & & & \\
Method IV & 59 & 1 & & $98 \%$ & $98 \%$ & $98 \%$ & $98 \%$ \\
& 1 & 59 & & & & & \\
\hline
\end{tabular}

to the Method I, the number of false detections (both FN and FP) are reduced, and the total accuracy is 97\%.

Method III. SVM with six vertical and horizontal features. To reduce the false detections (FN and FP), which are important parameters for a diagnosis system, three more features are extracted from the $\mathrm{ONH}$ in horizontal direction (Cup_H, Disc_H, and CDRh) and an SVM is used. Using the vertical and horizontal feature vectors, Method III gave a total accuracy of $99.2 \%$. Not only the accuracy increased, but also the FN error decreased to 0 . Comparing Method III to Method IV using a neural network (NN) with six features, an SVM decreases FN errors and performs with better accuracy than the NN technique. Table 2 shows a comparison 


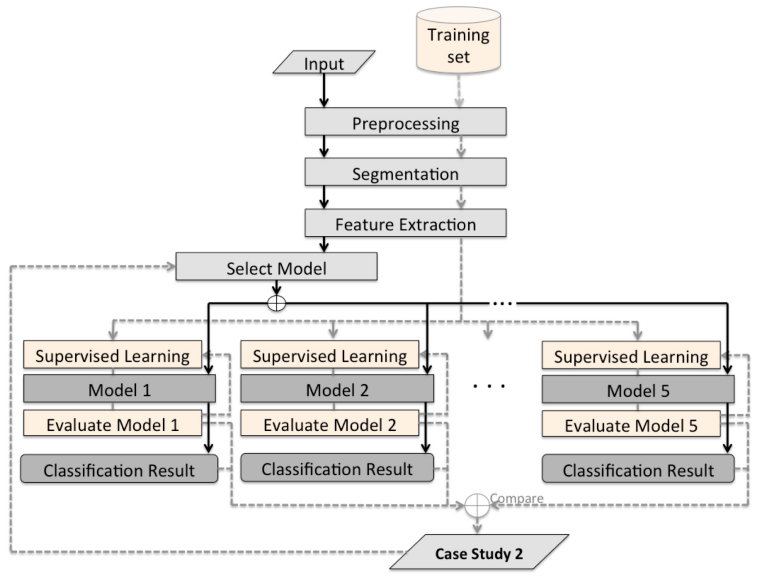

Fig. 9 Case study B.

of the classification performances among the three methods and the decreased number of errors (FP and FN). The confusion matrix is based on the structure in Table 1.

\section{Case study B}

As shown in Fig. 9, this case study proposes five models to distinguish three classes of glaucoma, non-glaucoma, glaucoma suspect, and glaucoma. The models are generated and the one that provides the highest performance to classify those three classes is selected. The OVR and UDT are applied in different ways to find a suitable classification model. The following vector shows a set of input features as used in the previous case study. Features $=[\mathrm{Cup} \mathrm{V}$, Disc_V, CDRv, Cup_H, Disc_H, CDRh].

When the features of the training data are fed to the model, machine learning generates the classifier model to diagnose the real input data. Based on the ground truth, Class 1 is given as healthy ${ }^{1-3,15}$ when CDRv is smaller than 0.3. Class 2 is suspected glaucoma, which has $\mathrm{CDRv}$ approximately between 0.3 and 0.6. The last class is glaucoma which has CDRv higher than 0.6. The following section explains the five models and compares their results.

Model I: OVR-SVM. This model consists of three submodules; OVR-SVM1, OVR-SVM2, and OVRSVM3 (Fig. 10). This technique separates one class from all the rest. The target is defined differently by setting 0 as target and 1 as the non-suspect class. OVR-SVM1, OVR-SVM2, OVR-SVM3 are set by labelling the normal, the glaucoma suspect, and the glaucoma as a target. As a result, the final class is chosen by selecting the highest score produced by each model. Table 3 provides a comparison of

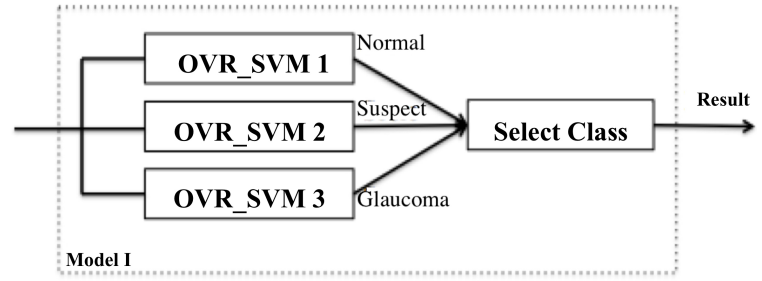

Fig. 10 Model I shows OVR-SVM.

Table 3 The confusion matrix and classification performances of each module for each model.

\begin{tabular}{|c|c|c|c|c|c|c|c|}
\hline \multirow[t]{2}{*}{ Model } & \multirow[t]{2}{*}{ Submodule } & \multicolumn{4}{|c|}{ Confusion matrix } & \multirow[t]{2}{*}{ SENS } & \multirow[t]{2}{*}{ PREC } \\
\hline & & $\mathrm{TP}$ & FP & $\mathrm{TN}$ & $\mathrm{FN}$ & & \\
\hline Iodel I & $\begin{array}{l}\text { OVR-SVM1 } \\
\text { OVR-SVM2 } \\
\text { OVR-SVM3 } \\
\text { Weighted av }\end{array}$ & $\begin{array}{l}60 \\
45 \\
59 \\
\text { erag }\end{array}$ & $\begin{array}{l}0 \\
5 \\
1\end{array}$ & $\begin{array}{l}95 \\
51 \\
85\end{array}$ & $\begin{array}{l}15 \\
69 \\
25\end{array}$ & $\begin{array}{l}80 \% \\
40 \% \\
70 \% \\
65 \%\end{array}$ & $\begin{array}{c}100 \% \\
90 \% \\
98 \% \\
96 \%\end{array}$ \\
\hline Model II & $\begin{array}{l}\text { UDT-SVM1 } \\
\text { UDT-SVM2 } \\
\text { Weighted av }\end{array}$ & $\begin{array}{l}60 \\
48 \\
\text { erag }\end{array}$ & $\begin{array}{l}0 \\
2\end{array}$ & $\begin{array}{l}95 \\
57\end{array}$ & $\begin{array}{c}15 \\
3\end{array}$ & $\begin{array}{l}80 \% \\
94 \% \\
90 \%\end{array}$ & $\begin{array}{c}100 \% \\
96 \% \\
97 \%\end{array}$ \\
\hline Model III & $\begin{array}{l}\text { UDT-SVM1 } \\
\text { UDT-SVM2 } \\
\text { Weighted av }\end{array}$ & $\begin{array}{l}45 \\
60 \\
\text { erag }\end{array}$ & $\begin{array}{l}5 \\
0\end{array}$ & $\begin{array}{l}51 \\
60\end{array}$ & $\begin{array}{c}69 \\
0\end{array}$ & $\begin{array}{c}40 \% \\
100 \% \\
82 \%\end{array}$ & $\begin{array}{c}90 \% \\
100 \% \\
97 \%\end{array}$ \\
\hline Model VI & $\begin{array}{l}\text { UDT-SVM1 } \\
\text { UDT-SVM2 } \\
\text { Weighted av }\end{array}$ & $\begin{array}{l}59 \\
57 \\
\text { erag }\end{array}$ & $\begin{array}{l}1 \\
3\end{array}$ & $\begin{array}{l}85 \\
50\end{array}$ & $\begin{array}{c}25 \\
0\end{array}$ & $\begin{array}{c}70 \% \\
100 \% \\
88 \%\end{array}$ & $\begin{array}{l}98 \% \\
95 \% \\
98 \%\end{array}$ \\
\hline Model V & $\begin{array}{l}\text { UDT-SVM1 } \\
\text { UDT-SVM2 } \\
\text { OVR-SVM1 } \\
\text { Weighted av }\end{array}$ & $\begin{array}{l}59 \\
50 \\
60 \\
\text { erag }\end{array}$ & $\begin{array}{l}1 \\
0 \\
0\end{array}$ & $\begin{array}{l}85 \\
57 \\
95\end{array}$ & $\begin{array}{c}25 \\
3 \\
15\end{array}$ & $\begin{array}{l}70 \% \\
94 \% \\
80 \% \\
81 \%\end{array}$ & $\begin{array}{c}98 \% \\
100 \% \\
100 \% \\
99 \%\end{array}$ \\
\hline
\end{tabular}

performances of each class.

Model II. UDT-SVM firstly classifies Class 1 and then Class 2 and Class 3. This model contains two submodules: UDT-SVM1 and UDT-SVM2 (Fig. 11). UDT-SVM1 is applied to identify healthy cases by labelling the healthy training target as 0 and the rest as 1 . The total number of UDT-SVM1's database is 170 . Then UDT-SVM2 is performed to classify

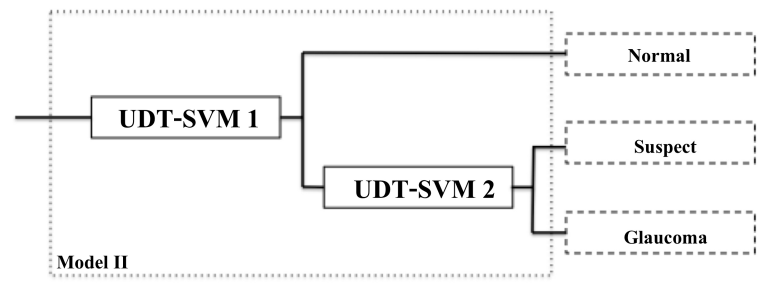

Fig. 11 Model II provides SVM with decision tree. 


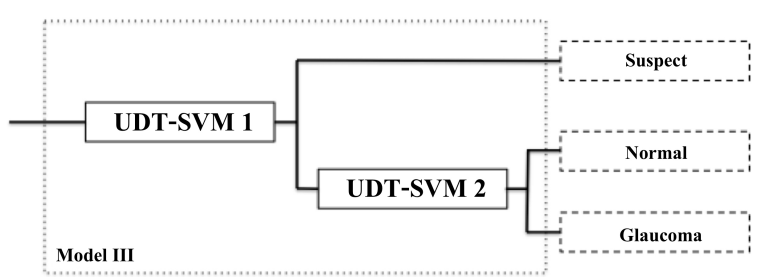

Fig. 12 Model III shows SVM with decision tree.

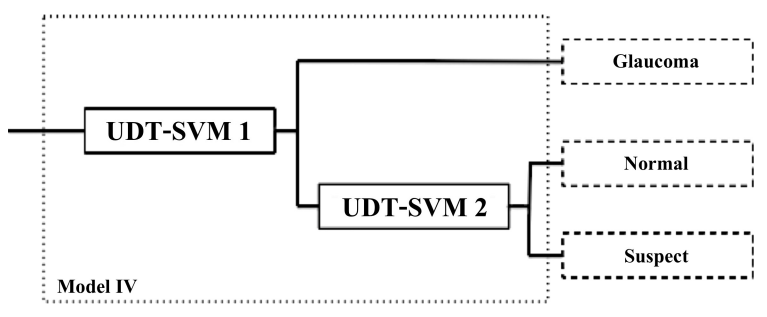

Fig. 13 Model IV shows SVM with decision tree.

between suspect and glaucoma classes. UDT-SVM1's dataset does not undergo the next classifier (UDTSVM2). The new quantity of database in UDT-SVM2 is 110 sets, and glaucoma suspect and glaucoma classes are classified by setting the glaucoma suspect class as 0 and glaucoma class as 1 . The result is shown in Table 3.

Model III. UDT-SVM firstly classifies Class 2 and then Class 1 and Class 3 (Fig. 12). In this model, the glaucoma suspect is classified first by UDT-SVM1. The glaucoma suspect class is set as 0 to be the target class. For UDT-SVM1, the total number of database is 170 . Then UDT-SVM2 is applied to classify normal and glaucoma classes. In this case, the dataset is reduced to be 110, which only belongs to normal and glaucoma classes. The normal class is set to 0 to become the target of UDT-SVM2. The result is shown in Table 3.

Model IV. UDT-SVM firstly classifies Class 3 and then Class 1 and Class 3. This model contains two submodules; UDT-SVM1 and UDT-SVM2. First, UDT-SVM1 is used to identify the glaucoma class. Thus the classifier target is set at 0 for the glaucoma class. UDT-SVM2 is then applied to classify normal and suspect classes (Fig. 13). The glaucoma data set is eliminated from UDT-SVM2 to 110 sets, the normal class is then labelled as 0 and suspect class as 1 . The result is shown in Table 3 .

Model V. UDT-SVM classifies Class 2 and Class 3 and OVR-SVM for Class 3. This model combines two techniques together that are UDT-SVM and OVRSVM (Fig. 14). The glaucoma class is classified by UDT-SVM1 as a first branch with 170 data sets. After

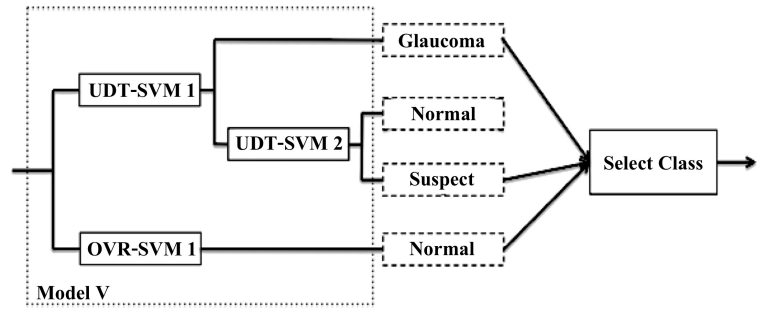

Fig. 14 Model V shows SVM with decision tree and oneversus-the-rest.

Table 4 The average precision of five models with polynomial kernel function.

\begin{tabular}{lcccc}
\hline Model & \multicolumn{3}{c}{ Precision } & PREC $_{\text {Avg }}$ \\
\cline { 2 - 4 } & Normal & Suspect & Glaucoma & \\
\hline Model I & $100 \%$ & $90 \%$ & $98 \%$ & $96 \%$ \\
Model II & $100 \%$ & $96 \%$ & $95 \%$ & $97 \%$ \\
Model III & $100 \%$ & $90 \%$ & $100 \%$ & $97 \%$ \\
Model IV & $95 \%$ & $100 \%$ & $98 \%$ & $98 \%$ \\
Model V & $100 \%$ & $100 \%$ & $98 \%$ & $99 \%$ \\
Model VI & $95 \%$ & $98 \%$ & $98 \%$ & $97 \%$ \\
\hline
\end{tabular}

eliminating glaucomatous data 60 sets, UDT-SVM2 is performed for the suspect class by setting to 0 the suspect datasets, and the output focuses only on the branch of the target class. At the same time, OVRSVM1 is performed by considering the normal class. The database of OVR-SVM1 contains all samples, 170 cases. Next, all candidates are analysed and selected for their belonging class. Finally, the result is shown in Table 3.

The PREC $_{\text {Avg }}$ in Table 4 is determined by averaging the weighted precisions of each class. Model VI uses an NN to classify the multi-class. It is shown that the false detection errors are 5,2 , and $2 \%$, respectively, from Class 1 to Class 3. However, for our proposed method, Model $\mathrm{V}$, the false detection errors are 0,0 , and $2 \%$. The errors from detecting glaucoma suspect are reduced which means that the early stage of glaucoma is robustly detected. By comparing all results in Table 4, Model V yields a better performance for the pre-screening of glaucoma suspect (Class 2) and glaucoma class (Class 3). Furthermore, Model V shows robustness to those two classes by a reduced number of false predictions of the target class from each submodule.

\section{DISCUSSION}

The experimental results from the previous part are discussed and concluded in this section. However, there are some limitations of this work when a pa- 
tient has an abnormal eye or has a risk for glaucoma without progression on the OD and OC. The nerve fibre layer around the $\mathrm{ONH}$, intraocular pressure inside the eyeball, vision field, and other risk factors must be analysed to diagnose this problem.

In Case study A, it is shown that threshold classification Method I by using CDRv is not adequate. There still occurs some misclassified data between 2 classes, glaucoma and non-glaucoma. The proposed technique includes an intelligent classification technique, which can adapt the decision boundary based on the training data. However, the performance of a classifier relies on the dimension of features. Important information needs to be detected to characterize the classes. The shape features are detected using image segmentation techniques and ellipse fitting to smoothen the data. The vertical sizes of the OC and OD are detected. Those features are applied to the classifier as proposed on Method II. The performances of the linear and polynomial kernel functions are compared. A suitable kernel function is selected based on the data types. A high number of features might affect the computational time of the system. Hence the less effective features are rejected. In Method III, six important features are used to differentiate the classes of glaucoma. The results indicate that Method I provided $80 \%$ accuracy. Method III, therefore, increased the classifying performance by adding three more horizontal features, it indicates that FN is reduced and ACC is increased to $99 \%$.

The Case study B concentrates on the comparison of five classifying models using a multi-class SVM. Each model is designed by combining different submodules using UDT and OVR techniques. Model I is robust to differentiate the characteristics of each class, and all databases are used to analyse and generate the decision function of each classifier. However, in Model I the number of submodules is equal to the number of the classification class, which is three submodules. The PREC are 100\% for Class 1, 90\% for Class 2, and 98\% for Class 3. The average PREC of Model I is $96 \%$, but the PREC for glaucoma suspect in Class 2 is $90 \%$, which means the remaining $10 \%$ is the false detection case. Thus Models II-V are analysed to decrease the false detections, especially for Class 2 and Class 3, which is an important target class to diagnose glaucoma. In this study, we emphasize the analysis that yields a reliable performance of the whole system.

For Models II-V combined with the UDT technique, it helps reduce the total computational time. The number of submodules in UDT is reduced to
2 compared to the OVR technique. Traditional UDT is not suitable for complicated conditions or data. However, the combination of UDT with SVM can overcome the limitations of binary classification SVM and the UDT-SVM and can classify the data with higher dimensions or complicated conditions. Next, we have to find a suitable determination of the node and branch separation. Models II-IV show the possible separation among three classes. Comparing Models II-IV, Model II has PREC 100\% for Class 1, 96\% for Class 2, and 95\% Class 3 with $97 \%$ $\mathrm{PREC}_{\text {Avg. }}$. It has high performance for Class 1, which means it can detect healthy cases $100 \%$ correctly, with 4\% and 5\% errors for glaucoma suspect and glaucoma cases. Model III has PERC 100\% for Class 1, $90 \%$ for Class 2, and $100 \%$ for Class 3 with $97 \% \mathrm{PREC}_{\mathrm{Avg}}$. The first terminal branch is the glaucoma suspect case, which is the class between normal and glaucoma classes. This model might not be adequate if the pattern of data points has some overlapping points from Class 1 and Class 3. Errors will occur more in Class 2 detection which is $10 \%$. Model IV is more robust for the suspect class, and glaucoma class with PREC 100\% and $98 \%$, respectively, and the $\mathrm{PREC}_{\mathrm{Avg}}$ is $98 \%$. However, to be more reliable, the false negative rate should be smaller. Based on our database from Mettapracharak hospital, Model V provided good performance among the other models. UDT-SVM2 helps in reducing the complicated training data from Class 3, then Class 2 can be detected easily. The overall PREC of Model V is 99\%, and this model is robust 100\% for Class 2 and $98 \%$ for Class 3, which are the target classes of this screening process. The target classes of this screening system are the classes that show abnormalities or suspected glaucoma. The healthy case is not the first priority target group of the screening system. The important target is to perform an efficient detection of glaucoma in early or advanced stages in order to prevent vision loss. The error detection from Class 2 and Class 3 is the considered problem because patients will not notice that they have abnormalities in their eyes. In the primary stage of glaucoma, there are also no early warning signs. Hence these two detected classes are necessary for helping preserve eyesight.

Acknowledgements: This study was financially supported by the Biomedical Engineering Research Unit (BIOMED), Sirindhorn International Institute of Technology (SIIT), National Science and Technology Development Agency (NSTDA), Japan Advanced Institute of Science and Technology (JAIST), Thammasat University, 
National Research University Project (NRU), and the Thailand Office of Higher Education Commission. The authors would also like to express our thanks towards Mettapracharak (Wat RaiKhing) Hospital for supporting us with medical advice and data sets.

\section{REFERENCES}

1. Kavitha S, Karthikeyan S, Duraiswamy K (2010) Early detection of glaucoma in retinal images using cup to disc ratio. In: International Conference on Computing Communication and Networking Technologies (ICCCNT), Karur, India, pp 1-5.

2. ManjulaSri R, Madhubabu C, Rao KMM (2013) LabVIEW based assessment of CDR for the detection of Glaucoma. In: International Conference on Emerging Trends in Computing, Communication and Nanotechnology (ICE-CCN), Tirunelveli, India, pp 683-6.

3. Agarwal A, Gulia S, Chaudhary S, Dutta MK, Travieso CM, Alonso-Hernandez JB (2015) A novel approach to detect glaucoma in retinal fundus images using cup-disk and rim-disk ratio. In: 4th International Work Conference on Bioinspired Intelligence (IWOBI), San Sebastian, Spain, pp 139-44.

4. Poshtyar A, Shanbehzadeh J, Ahmadieh H (2013) Automatic measurement of cup to disc ratio for diagnosis of glaucoma on retinal fundus images. In: 6th International Conference on Biomedical Engineering and Informatics (BMEI), Hangzhou, China, pp 24-7.

5. Lotankar M, Noronha K, Koti J (2015) Detection of optic cup and disc from color retinal images for automated diagnosis of glaucoma. In: IEEE UP Section Conference on Electrical Computer and Electronics (UPCON), Allahabad, India, pp 1-6.

6. Dutta MK, Mourya AK, Singh A, Parthasarathi M, Burget R, Riha K (2014) Glaucoma detection by segmenting the super pixels from fundus colour retinal images. In: International Conference on Medical Imaging, m-Health and Emerging Communication Systems (MedCom), Greater Noida, India, pp 86-90.

7. Cheng J, Liu J, Xu Y, Yin F, Wong DWK, Tan NM, Tao D, Cheng CY, Aung T, Wong TY (2013) Superpixel classification based optic disc and optic cup segmentation for glaucoma screening. IEEE Trans Med Imag 32, 1019-32.

8. Muramatsu C, Nakagawa T, Sawada A, Hatanaka Y, Hara T, Yamamoto Y, Fujita H (2011) Automated segmentation of optic disc region on retinal fundus photographs: comparison of contour modelling and pixel classification methods. Comput Meth Programs Biomed 101, 23-32.

9. Sedai S, Roy PK, Mahapatra D, Garnavi R (2016) Segmentation of optic disc and optic cup in retinal fundus images using shape regression. In: 38th Annual International Conference of the IEEE Engineering in Medicine and Biology Society (EMBS), Orlando, FL, pp 3260-4.
10. Joshi GD, Sivaswamy J, Krishnadas SR (2011) Optic disk and cup segmentation from monocular color retinal images for glaucoma assessment. IEEE Trans Med Imag 30, 1192-205.

11. Salam AA, Akram MU, Wazir K, Anwar SM, Majid M (2015) Autonomous glaucoma detection from fundus image using cup to disc ratio and hybrid features. In: IEEE International Symposium on Signal Processing and Information Technology (ISSPIT), Abu Dhabi, United Arab Emirates, pp 370-4.

12. Ayub J, Ahmad J, Muhammad J, Aziz L, Ayub S, Akram U, Basit I (2016) Glaucoma detection through optic disc and cup segmentation using Kmean clustering. In: International Conference on Computing, Electronic and Electrical Engineering (ICE Cube), Quetta, Pakistan, pp 143-7.

13. Xiong L, Li H, Zheng Y (2014) Automatic detection of glaucoma in retinal images. In: IEEE 9th Conference on Industrial Electronics and Applications (ICIEA), Hangzhou, China, pp 1016-9.

14. Naveen Kumar B, Chauhan RP, Dahiya N (2016) Detection of Glaucoma using image processing techniques: a review. In: International Conference on $\mathrm{Mi}$ croelectronics, Computing and Communications (MicroCom), Durgapur, India, pp 1-6.

15. Park J, Yae H (2002) Analysis of active feature selection in optic nerve data using labeled fuzzy C-mean clustering. In: Proceedings of the IEEE International Conference on Fuzzy Systems (FUZZ-IEEE), Honolulu, USA, pp 1580-5.

16. Chakravarty A, Sivaswamy J (2016) Glaucoma classification with a fusion of segmentation and imagedbased features. In: IEEE 13th International Symposium on Biomedical Imaging (ISBI), Prague, Czech Republic, pp 689-92.

17. Anusorn CB, Kongprawechnon W, Kondo T, Sintuwong S, Tungpimolrut K (2013) Image processing techniques for glaucoma detection using the cup-todisc ratio. Thammasat Int $J$ Sci Tech 18, 22-34.

18. Otsu N (1979) A threshold selection method from gray-level histograms. IEEE Trans Syst Man Cybern 9 , 62-6.

19. Kusumandari DE, Munandar A, Redhyka GG (2015) The component of GVF Snake Active Contour method and Ellipse Fit in optic disc detection for glaucoma diagnosis. In: International Conference on Automation, Cognitive Science, Optics, Micro Electro-Mechanical System, and Information Technology (ICACOMIT), Bandung, Indonesia, pp 123-6. 